\title{
Atopic Dermatitis Anti-IgE Paediatric Trial (ADAPT): the role of anti-lgE in severe paediatric eczema: study protocol for a randomised controlled trial
}

Susan Chan ${ }^{1,2^{*}}$ (D) Victoria Cornelius ${ }^{3}$, Tao Chen $^{4}$, Suzana Radulovic ${ }^{1,2}$, Mandy Wan ${ }^{1}$, Rahi Jahan ${ }^{1}$ and Gideon Lack ${ }^{2}$

\begin{abstract}
Background: The evidence for systemic treatments for severe childhood eczema is limited and largely based on extrapolation of data from adult studies. Current therapies are often immunosuppressant and may be associated with both short- and long-term side effects. There is increasing in vitro and murine-model evidence for the role of IgE in the immunopathogenesis of atopic eczema. The aim of the study is to assess whether anti-lgE treatment (omalizumab) improves eczema, compared to placebo.
\end{abstract}

Methods/design: The Atopic Dermatitis Anti-lgE Paediatric Trial (ADAPT) is a randomised, double-blind, placebo-controlled study assessing the role of anti-lgE in the management of severe paediatric eczema. Children with severe atopic eczema, with an objective SCORing Atopic Dermatitis (SCORAD) score of over 40 will be recruited. These children are candidates for systemic therapy, have failed systemic therapy or have experienced side effects from systemic therapy. Sixty-two patients aged between 4 and 19 years will receive anti-lgE for 6 months. The primary outcome measure will be the validated eczema score, the objective SCORAD at 24 weeks. This study has 90\% power to detect a $33 \%$ relative reduction in SCORAD between active and placebo groups, with 5\% significance.

Discussion: IgE may have a role to play in eczema, particularly in childhood. This forms the basis for the hypothesis that anti-lgE may be an effective treatment in this patient population.

This will be the largest study to evaluate the efficacy of anti-lgE (omalizumab) versus placebo in children with severe eczema. The findings will help to clarify the role of anti-lgE as a potential treatment option in patients with severe childhood eczema.

Trial registration: European Clinical Trials Database (EudraCT) Number: 2010-020841-29. Assigned on 14 May 2010. ISRCTN Registry, Identifier: ISRCTN15090567. Retrospectively assigned on 3 December 2014. ClinicalTrials.gov, Identifier: NCT02300701. First received 21 November 2014.

Keywords: Eczema, Paediatric, Atopic dermatitis, Anti-lgE, Omalizumab, Randomised controlled trial, Double blind, Placebo, Xolair ${ }^{\oplus}$

\footnotetext{
* Correspondence: susan.chan@kcl.ac.uk

'Guy's and St. Thomas' NHS Foundation Trust, Westminster Bridge Road, London SE1 7EH, UK

${ }^{2}$ King's College London, King's Health Partners, Asthma-UK Centre in Allergic

Mechanisms of Asthma, Department of Asthma, Allergy and Respiratory

Science, Guy's Hospital, London SE1 9RT, UK

Full list of author information is available at the end of the article
} 


\section{Background}

Atopic eczema (AE) (or atopic dermatitis) is an inflammatory, chronically relapsing, pruritic skin disease. The prevalence in the United Kingdom (UK) is $16 \%$ for 6 to 7-year-olds (with 2.2\% classed as severe) and 10.6\% for 13 to 14 -year-olds (with $1.5 \%$ classed as severe) [1]. AE is associated with a significant economic and psychosocial burden.

A complex interaction between genetics, environment and immunology define the pathophysiology of AE. There is increasing in vitro and murine-model evidence for the role of IgE in the immunopathogenesis of atopic dermatitis, with higher IgE levels linked with more severe disease $[2,3]$. IgE is likely to be of more relevance in paediatric disease than in adult disease, where $\mathrm{AE}$ is thought to become less allergen-driven and more 'autoreactive' [4]. This study focusses on a paediatric atopic population, to target patients in whom IgE is more likely to be relevant.

The management of $\mathrm{AE}$ includes the identification and elimination of trigger factors, appropriate use of topical treatments, and adequate patient education. When systemic therapy is required, systemic immunosuppressants, such as ciclosporin, azathioprine, and methotrexate, have been used [5]. However there is limited published evidence in childhood practice to guide clinical use. In addition, most of these drugs are not licensed for use in patients with severe AE in many countries. With the potential for serious side effects, these drugs are often not recommended for long-term use.

Omalizumab (Xolair ${ }^{\oplus}$, Novartis) is the only commercially available anti-IgE antibody. It binds to human IgE, limiting mast cell degranulation and inhibiting the release of inflammatory mediators. It is licensed in the European Union for use in chronic spontaneous urticaria from the age of 12 years, and severe persistent allergic asthma from the age of 6 years [6]. Safety data suggests that Xolair ${ }^{\circledR}$ (omalizumab) is well tolerated in children [7].

Our hypothesis is that anti-IgE will reduce the levels of IgE in children with severe $\mathrm{AE}$ and alleviate their symptoms.

\section{Objectives}

Primary objectives

1. To determine whether the intervention is associated with an improvement in eczema severity, compared to placebo.

\section{Secondary objectives}

1. To evaluate whether the intervention is associated with a change in eczema severity, eczema quality of life and coexisting allergic disease
2. To assess whether it is safe and well tolerated in eczema

3. To investigate whether the intervention is associated with fewer eczema exacerbations and infective episodes of eczema

4. To determine the change in reactivity to allergens

\section{Methods/design}

\section{Study design}

The Atopic Dermatitis Anti-IgE Paediatric Trial (ADAPT) is a randomised, double-blind, placebo-controlled study. It uses a hub and spoke method of recruitment where treatment will be delivered at a single centre, but recruitment will occur from hospitals in and around London, United Kingdom. The trial will test the superiority of anti-IgE (omalizumab) versus placebo in the treatment of severe childhood eczema. The protocol was designed in accordance with the SPIRIT (Standard Protocol Items: Recommendations for Interventional Trials) guidelines for interventional trials (Additional file 1: Figure S1; and Additional file 2: Figure S2).

Sixty-two children, aged 4-19 years, with severe eczema will be enrolled in the study which is based at Guy's and St. Thomas' NHS Foundation Trust, London, United Kingdom.

\section{Study population}

The study population will be children with severe AE, who are candidates for systemic therapy for AE. Children will also be eligible if they have failed systemic therapy, or have experienced side effects from systemic therapy.

\section{Inclusion criteria}

1. Aged 4-19 years

2. Severe eczema with (1) an objective SCORing Atopic Dermatitis (SCORAD, a validated eczema severity score) score of over 40 , which is (2) unresponsive to optimal topical therapy (potent topical steroids and/or topical calcineurin inhibitors) or systemic therapy, with (3) no impression of lack of compliance, (4) a (Children's) Dermatology Life Quality Index Questionnaire ((C)DLQI) score of $\geq 10$ and (5) where active skin infection has been ruled out and/or adequately treated

3. A raised specific IgE (SpIgE) (>0.35 IU/ml or $\mathrm{kUA} / \mathrm{L})$ or skin-prick test (SPT) (>3 mm) to at least one food allergen or one aeroallergen and/or

4. The clinical impression that allergic exposures cause worsening eczema

5. Total IgE level $>300 \mathrm{IU} / \mathrm{ml}$ or $\mathrm{kU} / \mathrm{L}$

6. Clinically proven IgE-mediated allergic disease including at least one of the following: (1) immediate hypersensitivity to a food as proven by raised SpIgE or SPT greater than the $95 \%$ positive predictive value 
or $\geq 8 \mathrm{~mm}$, or a positive double-blind, placebocontrolled food challenge, (2)

allergic rhinoconjunctivitis as defined by sensitisation to a respiratory allergen and a clinical history of rhinoconjunctivitis symptoms when exposed to the relevant allergen, (3) allergic asthma: a history of a cough, wheeze or shortness of breath that (a) was responsive to therapy with bronchodilators on two or more occasions in the previous 24 months, (b) required one visit to a physician in the previous 24 months and (c) occurred during the night, during early morning or upon exercising in the intervals between exacerbations at any time in the previous 12 months and (d) where allergic exacerbations can be clinically related to an allergen exposure with a corresponding positive SPT or SpIgE to the allergen

7. Written informed consent to participate, and assent if appropriate

\section{Exclusion criteria}

Children will not be able to participate if (1) they and/or their families are unable to comply with the regime of two to four weekly injections and clinic visits, (2) there is evidence of underlying immune compromise, autoimmune disease, immune complex-mediated conditions, (3) there is malignancy or a history of malignancy, (4) there is a known cardiovascular or ischaemic cerebrovascular abnormality, (5) there is other serious or uncontrolled systemic disease, (6) the subject is pregnant or lactating, (7) there is a known history of hypersensitivity or anaphylaxis to anti-IgE injections or its constituents; (8) there is insufficient understanding of the trial assessments, (9) they have participated in a clinical trial of an investigational medicinal product (CTIMP) in the previous 60 days or (if known) four half-lives of the medication under investigation, whichever is the greater. In this case, entry may be delayed until the appropriate time or (10) the investigator feels that there is a good clinical reason why the child would be unsuitable for the study.

\section{Recruitment}

Sixty-two children with severe AE, will be enrolled into the study which is based at Guy's and St. Thomas' NHS Foundation Trust. A hub and spoke method of recruitment will be employed. Referring hospitals from in and around London will be able to refer patients to the study team. Recruitment will be open to participants in the UK who are willing to travel to the central treatment centre for their treatment and follow-up visits.

\section{Intervention}

Each participant will be enrolled for 48 weeks, consisting of a 24-week treatment period and a 24-week follow-up period. A detailed timeline of the study is shown in the Gantt chart in Additional file 3: Figure S3.

The active treatment is omalizumab (Xolair ${ }^{\circ}$ ) injection, manufactured by Novartis and provided as 150-mg single-use vial. The manufacturer also provided the comparator. Placebo is formulated to be comparable in appearance to the omalizumab and contains all of the same excipients except the drug substance. The dose and dosing frequency of omalizumab (Xolair ${ }^{\circ}$ ) or placebo will be determined by baseline total IgE (range: 30 to $1500 \mathrm{IU} / \mathrm{ml}$ ), measured before treatment commences, and body weight $(\mathrm{kg})$ at the randomisation visit. The dose of omalizumab (Xolair ${ }^{\circ}$ ) will be calculated using the latest manufacturer's dosing tables (according to the current Summary of Product Characteristics) [8]. The dose of Xolair (omalizumab) stated on the table, closest to that child's weight and IgE levels, is used. Doses are $75,150,225,300,450,525$ or $600 \mathrm{mg}$ every 2 or 4 weeks. The equivalent volume for placebo doses will be calculated in the same way. Participants will remain on the same dose throughout the 24 weeks of treatment. Treatment is administered subcutaneously in the deltoid region or the thigh as one to four injections depending on the dose. Anaesthesia using topical local anaesthetic (lidocaine cream) or ethyl chloride spray can be used prior to the injection, according to patient preference.

\section{Randomisation and blinding}

Participants who consent to participate and who fulfil the eligibility criteria are randomly allocated to receive either omalizumab (Xolair ${ }^{\circ}$ ) or placebo in a 1:1 allocation ratio using a secure web-based randomisation system. The allocation sequence is computer-generated by the UK Clinical Research Collaboration (UKCRC)registered King's College London Clinical Trials Unit in conjunction with an independent statistician. Participants are allocated to treatment group using minimisation with variables $\operatorname{IgE}(\leq 1500,>1500 \mathrm{IU} / \mathrm{ml})$ and age (below 10 years or 10 years and older).

Blinding is maintained by strictly adhering to established procedures to maintain separation between staff who take outcome measurements and staff who deliver the treatment. All study team physicians, researchers and research nurses involved in primary outcome assessments, as well as participants and their families, are blinded to treatment allocation until the primary analysis is completed. To ensure that these individuals remain blinded to treatment allocation, randomisation details are sent electronically to the independent pharmacy team, and the preparation and administration of the treatment will be restricted to a group of trained clinical staff. Unblinded clinical staff are assigned to collect and 
then return used vials of active or placebo to pharmacy. Preparation and administration of treatment by unblinded staff will occur in a closed treatment room, separate from the main clinical area. Entry to this room is barred to blinded staff during the preparation and administration of the treatment by unblinded staff. Unblinded clinical staff will not be involved in trial-related primary outcome assessments to minimise the risk of disclosing the allocation of treatment. Staff members who obtain outcome measurements are not involved in any of the steps involving handling of the intervention.

All patients will be provided with an emergency card with relevant contact details to be carried for the duration of the trial. Code break will be provided in clinically relevant situations to clinicians, by the pharmacy department.

The study statistician will analyse the data blinded to treatment allocation.

\section{Concomitant medications}

Participants may continue to take their conventional treatments for $\mathrm{AE}$ during the course of the study. Concomitant $\mathrm{AE}$ medications would include topical treatments including any emollients, bath additives, topical steroids, topical calcineurin inhibitors, wet wraps and systemic treatment.

During treatment with omalizumab (Xolair $\left.{ }^{\circ}\right) /$ placebo, the participants/parents will monitor the child's AE at home and, if there is any deterioration, this would trigger the participants/parents to contact the study team to discuss drug doses or a reassessment and modification of therapy. An exacerbation will be identified and managed, and any additional therapy or changes in doses of existing treatment will be recorded.

Any medication required for any ongoing illness, contraception and any rescue medications will also be permitted and recorded. Female patients who have attained menarche will undergo a pregnancy test at the baseline and randomisation visits.

\section{Outcomes}

Details of the primary and secondary outcomes of the study are summarised in Additional file 4: Table S1.

\section{Primary outcome \\ Objective SCORAD}

This will be based upon the assessment of the objective SCORAD (SCORing Atopic Dermatitis) score after 24 weeks of treatment. Further assessments at 36 and 48 weeks (post-treatment reviews) will be performed.

The objective SCORing Atopic Dermatitis (eczema severity score) is a validated clinical tool used to assess the extent and severity of eczema [9]. It evaluates six clinical characteristics to determine disease severity: (1) erythema, (2) edema/papulation, (3) oozing/crust, (4) excoriation, (5) lichenification and (6) dryness. The maximum score is 83 .

\section{Secondary outcomes \\ Treatment failure}

Treatment failure will be defined in patients who, after the first 12 weeks of treatment, have persistent severe eczema despite two courses of rescue therapy with oral prednisolone $(0.5$ to $1 \mathrm{mg} / \mathrm{kg} /$ day of oral prednisolone for a week at a maximum dose of $40 \mathrm{mg} /$ day, followed by a week at $50 \%$ of this dose).

\section{Alternative systemic therapy}

An assessment will be made of patients in whom (1) alternative systemic therapy has been started as a result of treatment failure as defined above or (2) where alternative systemic therapy is started after 12 weeks, and by 30 weeks.

\section{Eczema quality of life}

This will be assessed by the Patient-oriented Eczema Measure Questionnaire (POEM) and the (Children's) Dermatology Life Quality Index Questionnaire (C)DLQI.

The POEM is a validated, patient-derived assessment measure for monitoring atopic eczema severity over the past week [10]. The questionnaire has seven items, each with a 5-point scale from no days to every day. The scores range from 0 to 28 . The data will be collected at baseline, 4-weekly during treatment up to 24 weeks, and at 36 and 48 weeks.

The (C)DLQI is a validated questionnaire designed to measure the condition of the participant's skin over the past week [11]. The questionnaire has 10 questions and the total score ranges from 0 to 30 [12]. The information will be followed up at baseline, 4-weekly during treatment up to 24 weeks, and at 36 and 48 weeks.

\section{Eczema severity}

This will be assessed by the objective and subjective SCORAD scores, and the Eczema Area and Severity Index (EASI).

The subjective SCORAD score is an add-on score to the objective SCORAD score, which also assesses subjective symptoms of pruritus and sleep loss [13], each on a scale of 0 to 10 [13]. The subjective score adds up to an additional 20 points to the objective SCORAD score.

The EASI is an investigator-assessed instrument to measure the extent (area) and severity of atopic eczema [14]. The severity of four features (erythema, edema/ papulation, excoriation and lichenification) are scored from 0 to 3 (none, mild, moderate and severe, respectively) with half-steps allowed, in a representative patch of eczema in four regions (head/neck, upper limbs, trunk, lower limbs). The percentage area affected is 
calculated. The EASI scores range from 0 to 72 , and higher scores represent more severe eczema.

\section{Effect on coexisting allergic disease}

This will be assessed by the Paediatric Allergic Disease Quality of Life Questionnaire (PADQLQ), a measure of health-related quality of life that encompasses effects of allergic conditions on a child's or teenager's eyes, ears, nose, lungs, skin, emotions, and everyday activities over the past week [15]. The PADQLQ contains 26 questions, with a 7-point scale from 'not troubled' to 'extremely troubled'.

\section{Number of eczema exacerbations}

The number of eczema exacerbations will be recorded. Exacerbations will be defined as clinician-diagnosed exacerbation of eczema or increase in the SCORAD score by 15 points from the last recorded SCORAD score with patient/parent/guardian perception of worsening eczema.

\section{Infective episodes of eczema}

Infective episodes of eczema will be defined as cliniciandiagnosed and treated infective episode of eczema, or clinically apparent, culture-positive infective exacerbations.

\section{Total and allergen-specific IgE (SplgE)}

This will be determined at screening and at 24 weeks.

\section{Reactivity to food and aeroallergens}

The SPT is an assessment of the allergic response to specific food and aeroallergens. The SPT introduces a tiny amount of allergen into the skin, eliciting a small, localised allergic response in the form of a wheal and flare at the site of testing $[16,17]$.

\section{Steroid cream usage}

The amount of potent steroid creams and calcineurin inhibitors used will be assessed. Tubes of creams are weighed at each visit using digital scales. The frequency of use per day and per week since the last visit, and the body surface area covered, will be recorded.

\section{Data collection and management}

Data will be collected on source data worksheets and stored in the patient's medical notes, and will be transcribed to the electronic Case Report Form (eCRF) system. The eCRF will be designed and maintained by the King's Clinical Trials Unit using the InferMed MACRO system (Version 4.0), which is compliant with Good Clinical Practice (GCP) and 21 CRF Part 11. At the end of the study, the eCRF system will be locked and data exported for final analysis. Patient data will be anonymised. All anonymised data will be stored on a password-protected computer. All trial data will be stored and archived in line with the Medicines for Human Use (Clinical Trials) Amended Regulations 2006 as defined in the King's Health Partners Clinical Trials Office Archiving Standard Operating Procedure.

\section{Statistics \\ Sample size}

Omalizumab is administered at 2- to 4-weekly intervals, by subcutaneous injection, at a suitable health care facility. It is available in the UK under a negotiated patient access scheme for asthma and chronic urticaria. A reasonable treatment benefit would be required for omalizumab to be adopted into practice. We aim to detect a $33 \%$ relative reduction in SCORAD scores. In order to detect a change in the SCORAD score of 13.5 points between the treatment arms, assuming an SD of 15 [18] with $5 \%$ significance and $90 \%$ power, and incorporating a $15 \%$ dropout rate, we aim to recruit 62 participants (31 to each arm).

\section{Analysis}

A Consolidated Standards of Reporting Trials (CONSORT) flow chart will be constructed and will include the number of eligible patients, number of patients agreeing to enter the trial, number of participants withdrawing and the number included in the analyses [19].

Baseline characteristics will be summarised and tabulated by treatment arm. The analysis will follow an intention-to-treat (ITT) principle and participants will be analysed in the arm to which they were allocated regardless of subsequent treatment received. The number lost to follow-up will be tabulated by treatment arm and visit. The proportions of participants missing objective SCORAD values will be summarised in each arm and at each time point. The baseline characteristics (age, gender, objective and subjective SCORAD, Body Mass Index (BMI), asthma $(\mathrm{Y} / \mathrm{N})$, food allergy $(\mathrm{Y} / \mathrm{N})$, rhinoconjunctivitis $(\mathrm{Y} / \mathrm{N})$ and referral source (self-referred/tertiary) of those missing follow-up will be compared to those with complete follow-up. The recorded reasons for withdrawal from the trial will be summarised [20].

A linear mixed model will be used to obtain an estimate for the mean difference in objective SCORAD scores between the two treatment groups. Participant will be included as a random intercept (investigating adding a random slope on time), time (investigating the possibility of linearising this effect across 8, 12, 16, 20 and 24 weeks), time-by-group interaction, baseline objective SCORAD score, $\operatorname{IgE}(\leq 1500,>1500 \mathrm{IU} / \mathrm{ml})$ and age (below 10 years or 10 years and older) as fixed effect. An overall treatment effect for the objective SCORAD score at 24 weeks will be estimated. A number of sensitivity analyses will be performed to estimate the treatment effect adjusting for use of topical steroids and 
alternative systemic therapy. A complier average causal effect (CACE) will be performed where 'complier' will be defined as a participant who completes more than $50 \%$ of injections [21].

Secondary analysis will be based on the ITT population and primarily focussed on the outcome at week 24 . Subjective SCORAD scores will be analysed in a similar manner as for objective SCORAD scores. For all other outcomes we will only adjust for the minimisation variables $\operatorname{IgE}(\leq 1500$, $>1500 \mathrm{IU} / \mathrm{ml}$ ), age (below 10 years or 10 years and older) and baseline data (as appropriate). Binary outcomes will be analysed using a logistic regression model and count outcomes using Poisson regression, negative binomial regression or zero-inflated Poisson, as appropriate, and continuous outcomes using a linear regression model.

Adverse events will be tabulated separately by type (adverse events, adverse reaction, unexpected adverse reaction, serious adverse event, serious adverse reaction or unexpected serious adverse reactions). These will be summarised overall (0-48 weeks) and the time of occurrence will be explored by randomised intervention where appropriate.

A detailed statistical analysis plan has been developed in conjunction with the protocol and is available [Reference from Trials if accepted].

\section{Discussion}

The management of AE necessitating systemic therapy is currently an area of intense interest. When systemic therapy is required in childhood $\mathrm{AE}$, systemic immunosuppressants have been used with limited evidence, and in many cases as an unlicensed indication [5].

There is increasing evidence for the role of IgE in the immunopathogenesis in AE. Omalizumab (Xolair, Novartis) is the only commercially available anti-IgE antibody. ADAPT is the largest randomised, double-blind, placebocontrolled study to date evaluating anti-IgE (omalizumab) in severe childhood AE. The aim is to definitively assess the role of anti-IgE as an alternative systemic treatment for AE.

\section{Trial status}

The ADAPT study received favourable ethical opinion on 7 July 2011 and local R\&D approvals were obtained on 11 November 2014. Participant recruitment started on 20 November 2014 and is ongoing.

\section{Additional files}

Additional file 1: Figure S1. SPIRIT 2013 Checklist. (DOC 272 kb)

Additional file 2: Figure S2. Schedule of enrolment, interventions, and assessments. (DOC $68 \mathrm{~kb}$ )

Additional file 3: Figure S3. Gantt chart (timeline of the study, DOC). (DOCX $16 \mathrm{~kb}$ )

Additional file 4: Table S1. Primary and secondary outcomes (primary and secondary outcome measures of the study). (DOCX $20 \mathrm{~kb}$ )

\section{Abbreviations}

(C)DLQI: (Children's) Dermatology Life Quality Index; AD: Atopic dermatitis; ADAPT: Atopic Dermatitis Anti-IgE Paediatric Trial; AE: Atopic Eczema; CTIMP: Clinical trial of an investigational medicinal product; EASI: Eczema Area and Severity Index; eCRF: Electronic Case Report Form; IgE: Immunoglobulin E; PADQLQ: Paediatric Allergic Disease Quality of Life Questionnaire; POEM: Patient Oriented Eczema Measure; REC: Research Ethics Committee; SCORAD: SCORing Atopic Dermatitis; SPT: Skin-prick test

\section{Acknowledgements}

The study is supported by the NIHR Biomedical Research Centre, which is based at Guy's and St. Thomas' NHS Foundation Trust and King's College London, and by a National Institute for Health Research comprehensive Biomedical Research Centre award to Guy's and St. Thomas' NHS Foundation Trust and King's College London. The authors would like to thank all the participants and health care professionals for their involvement in the study. The authors would also like to thank Novartis for providing the Investigational Medicinal Product (IMP), the United Kingdom Clinical Research Collaboration-registered King's Clinical Trials Unit at King's Health Partners, and the National Eczema Society for their support.

A special thank you also to the all the staff involved in the ADAPT study: Muhsinah Adam, Freda Adjei, Aikaterini Anagnostou, David Atherton, Emily Banks, Kathryn Brighthouse, Helen Brough, Natalia Cartledge, Richard Cleaver, Louise Coverdale, Mary De Sousa, George Du Toit, Helen Fisher, Joanna Gambell, Erika Harnik, Claire Hearn, Jemma Hodges, Christine Jameson, Patricia Kane, Colm Keenan, Katherine Knight, Marta Krawiec, Melissa Llewellyn, Tom Marrs, Gary McGreevy, Una O’Dwyer-Leeson, Victoria Offord, Devi Patkunam, Ewa Pietraszewicz, Gemma Scanlan, Alick Stephens, Kate Swan, Prabalini Thaventhiran, Victoria Timms, Victor Turcanu, Rosy Wells, Charlotte Walker, Fiona Watson, Emma Wedgeworth and Louise Young. We thank the Data Monitoring and Ethics Committee (Professor Graham Roberts (Chair), Dr. Ahmed Massoud, Ms. Helen Moyses) and the Trial Steering Committee (Dr. Nerys Roberts (Chair), Dr. Mike Coren, Dr. Helen Cox, Professor John Harper, Mrs. Kathryn Humphreys, Dr. Susan Chan, Dr. Emma Wedgeworth, Professor Gideon Lack, Dr. Victoria Cornelius) for their advice and contributions to the protocol and statistical analysis plan. We also wish to thank Kun Liu for his important contribution to the development of the statistical methods.

\section{Funding}

This Investigator-led study is funded by the National Institute for Health Research (NIHR) Efficacy and Mechanism Evaluation (EME) Programme (NIHR EME 11/14/24) and Guy's and St. Thomas' Charity (Reference number: R090777). IMP and placebo were provided by Novartis. The study was designed and delivered in collaboration with the UK Clinical Research Collaboration (UKCRC)-registered King's College London Clinical Trials Unit. The views expressed in this article are those of the authors and do not necessarily reflect those of the NIHR, EME Programme, the NHS or the Department of Health.

\section{Availability of data and materials \\ Not applicable.}

\section{Authors' contributions}

SC is the chief investigator, obtained grant funding, conceived and designed the study and drafted the manuscript. VC contributed to the design of the study, designed the statistical analysis plan and reviewed the manuscript. TC contributed to the design of the statistical analysis plan, and reviewed the manuscript. SR reviewed the manuscript. MW reviewed the manuscript. RJ managed the study and drafted the manuscript. GL obtained grant funding, conceived and designed the study, and reviewed the manuscript. All authors provided final approval of the manuscript.

\section{Competing interests}

The authors declare that they have no competing interests.

\section{Consent for publication}

The Participant Information Sheet and Participant Consent Form, which have been approved by the REC, inform participants and their families that the results will be submitted for publication in a scientific journal. Participants will not be identifiable in any publications

The results will be disseminated via presentations to societies and publication in peer-reviewed journals. 


\section{Ethics approval and consent to participate}

The trial will be conducted in compliance with the principles of the Declaration of Helsinki (1996), the principles of GCP and all of the applicable regulatory requirements. Regulatory approval has been sought from the Medicines and Healthcare products Regulatory Agency (MHRA). The King's Health Partners Clinical Trials Office (KHPCTO) will manage the sponsor's responsibilities and quality assurance to ensure compliance with the Clinical Trial Regulations. Ethical approval is overseen by London Westminster NHS Research Ethics Committee (REC reference number: 11/LO/0123). Written informed consent will be obtained from all participants before any study procedure is performed. The participant (and/or parent/guardian), will have the opportunity to review the Participant Information Sheet and Participant Consent Form, and agree that they fully understand the details of the study procedures. Informed consent will be taken by a suitably qualified and experienced individual who has been delegated this duty by the principal investigator.

\section{Author details}

'Guy's and St. Thomas' NHS Foundation Trust, Westminster Bridge Road, London SE1 7EH, UK. 'King's College London, King's Health Partners, Asthma-UK Centre in Allergic Mechanisms of Asthma, Department of Asthma, Allergy and Respiratory Science, Guy's Hospital, London SE1 9RT, UK. ${ }^{3}$ Imperial Clinical Trials Unit, School of Public Health, Imperial College London, Stadium House, 68 Wood Lane, London W12 7RH, UK. ${ }^{4}$ Department of Clinical Sciences, Liverpool School of Tropical Medicine, Liverpool L3 5QA, UK.

Received: 2 November 2016 Accepted: 19 January 2017

Published online: 22 March 2017

\section{References}

1. Williams $\mathrm{H}$, et al. Is eczema really on the increase worldwide? J Allergy Clin Immunol. 2008;121(4):947-54.

2. Laske N, Niggemann B. Does the severity of atopic dermatitis correlate with serum lgE levels? Pediatr Allergy Immunol. 2004:15(1):86-8.

3. Du Toit $\mathrm{G}$, et al. Identifying infants at high risk of peanut allergy: the Learning Early About Peanut Allergy (LEAP) screening study. J Allergy Clin Immunol. 2013;131(1):135-43. e1-12.

4. Bieber T. Atopic dermatitis. N Engl J Med. 2008:358(14):1483-94.

5. Proudfoot LE, et al. The European TREatment of severe Atopic eczema in children Taskforce (TREAT) survey. Br J Dermatol. 2013;169(4):901-9.

6. Omalizumab for treating severe persistent allergic asthma: asthma (severe, persistent, patients aged 6+, adults) - omalizumab (replaces TA133 and TA201). NICE technology appraisal guidance (TA278); 2013. https://www nice.org.uk/guidance/ta278.

7. Milgrom $\mathrm{H}$, et al. Safety of omalizumab in children with inadequately controlled moderate to severe allergic (lgE-mediated) asthma. Am J Respir Crit Care Med. 179;2009:A2809.

8. Summary of Product Characteristics - $150 \mathrm{mg}$ powder and solvent solution for injection. Novartis Pharmaceuticals UK Ltd; 2009. https://www.medicines. org.uk/emc/medicine/24912.

9. European Task Force on Atopic Dermatitis. Severity scoring of atopic dermatitis: the SCORAD index. Consensus Report of the European Task Force on Atopic Dermatitis. Dermatology. 1993;186(1):23-31.

10. Charman CR, Venn AJ, Williams HC. The patient-oriented eczema measure: development and initial validation of a new tool for measuring atopic eczema severity from the patients' perspective. Arch Dermatol. 2004;140(12):1513-9.

11. Lewis-Jones MS, Finlay AY. The Children's Dermatology Life Quality Index (CDLQI): initial validation and practical use. Br J Dermatol. 1995;132(6):942-9.

12. Holme SA, et al. The Children's Dermatology Life Quality Index: validation of the cartoon version. Br J Dermatol. 2003;148(2):285-90.

13. Kunz B, et al. Clinical validation and guidelines for the SCORAD index: consensus report of the European Task Force on Atopic Dermatitis. Dermatology. 1997;195(1):10-9.

14. Hanifin JM, et al. The eczema area and severity index (EASI): assessment of reliability in atopic dermatitis. EASI Evaluator Group Exp Dermatol. 2001; 10(1):11-8.

15. Roberts G, Hurley C, Lack G. Development of a quality-of-life assessment for the allergic child or teenager with multisystem allergic disease. J Allergy Clin Immunol. 2003;111(3):491-7.
16. King R, et al. Paediatric Skin Prick Testing: standary operating procedure. British Society for Allergy and Clinical Immunology; 2010. https://www.bsaci. org/_literature_121183/Paediatric_skin_prick_testing_guideline.

17. Heinzerling $L$, et al. The skin prick test-European standards. Clin Trans Allergy. 2013;3(1):3.

18. Hindley $D$, et al. A randomised study of 'wet wraps' versus conventional treatment for atopic eczema. Arch Dis Child. 2006;91(2):164-8.

19. Schulz KF, Altman DG, Moher D. CONSORT 2010 statement: updated guidelines for reporting parallel group randomised trials. BMJ. 2010;340:c332.

20. Royston P. Multiple imputation of missing values: update of ice. Stata J. 2005:5(4):527-36.

21. Sussman JB, Hayward RA. An IV for the RCT: using instrumental variables to adjust for treatment contamination in randomised controlled trials. BMJ. 2010;340:c2073

\section{Submit your next manuscript to BioMed Central and we will help you at every step:}

- We accept pre-submission inquiries

- Our selector tool helps you to find the most relevant journal

- We provide round the clock customer support

- Convenient online submission

- Thorough peer review

- Inclusion in PubMed and all major indexing services

- Maximum visibility for your research

Submit your manuscript at www.biomedcentral.com/submit
) BioMed Central 